\title{
NOUVELle
}

Le réseau cérébral

fonctionnel des enfants atteints d'autisme

\section{Redondance et déconnexion}

Maxime Taquet ${ }^{1,2}$, Jurriaan M. Peters ${ }^{1,3}$
${ }^{1}$ Computational radiology laboratory,

Boston children's hospital,

Harvard medical school, Boston, États-Unis ;

${ }^{2}$ ICTEAM institute, université catholique de Louvain,

Louvain-la-Neuve, Belgique;

${ }^{3}$ Department of Neurology and the Division of Epilepsy and Clinical Neurophysiology, Boston Children's Hospital, Harvard Medical School, Boston, États-Unis.

maxime.taquet@childrens.harvard.edu

jurriaan.peters@childrens.harvard.edu
> Les troubles du spectre autistique (TSA) sont un syndrome comportemental dont les facteurs de risques sont complexes. Selon les dernières estimations, le taux de prévalence de l'autisme est de plus de 60 enfants sur 10000 en France et aurait triplé en 10 ans [1]. Malgré cette prévalence et les lourdes conséquences pour la santé publique, les mécanismes neurobiologiques de l'autisme restent largement inconnus.

\section{Modèle neurobiologique}

de l'autisme : hypothèse et complexité méthodologique

Une hypothèse récente présente l'autisme comme un syndrome de déconnexion par lequel le déficit fonctionnel serait lié à une intégration altérée des signaux émanant de régions distantes du cerveau. Différentes études ont appuyé ce modèle [2], mais des problèmes méthodologiques et des conclusions contradictoires ont mis en cause sa validité [3].

Par ailleurs, l’hétérogénéité étiologique et clinique de l'autisme rend plus complexes la détection et la caractérisation de mécanismes neurobiologiques communs aux troubles du spectre autistique $[4,5]$. Traditionnellement, les recherches se sont focalisées sur l'autisme non syndromique, empêchant la généralisation des conclusions à l'ensemble du spectre autistique, quelle que soit l'étiologie.

La sclérose tubéreuse de Bourneville : une « fenêtre » pour étudier l'autisme La sclérose tubéreuse de Bourneville (STB) est un syndrome génétique neurocutané dont les manifestations peuvent avoir des répercussions neurologiques désastreuses. La STB est l'une des causes les mieux établies d'autisme (environ $40 \%$ des patients développent un trouble du spectre autistique). Cette incidence épidémiologique en fait une condition idéale pour étudier l'autisme syndromique [5]. Une récente étude a mis en évidence des différences de connectivité dans le cerveau des patients atteints d'autisme [4]. Pour la première fois, cette étude intégrait à la fois des patients atteints d'autisme non syndromique ( $n=16$ ) et des patients dont l'autisme est lié à la STB $(n=14)$. La connectivité cérébrale chez ces patients a été comparée à deux groupes contrôles: un groupe de volontaires neurotypiques $(n=46)$ et un groupe de patients STB non atteints d'autisme $(n=29)$. Le choix de ces populations a permis d'identifier des mécanismes neurobiologiques communs à l'autisme syndromique et non syndromique (Figure $1 A$ ).

\section{Mesurer la connectivité cérébrale}

\section{à partir d'images médicales}

Le cerveau est constitué de différentes populations neuronales interconnectées. Les connexions principales entre ces populations neuronales peuvent être détectées et caractérisées par l'imagerie médicale. L'imagerie pondérée en diffusion, par exemple, permet de détecter les faisceaux de fibres de la matière blanche et, partant, de caractériser la connectivité structurelle du cerveau [8].

Pour comprendre comment la fonction neurophysiologique est reliée à cette architecture, la connectivité fonctionnelle doit aussi être analysée. La connectivité fonctionnelle entre deux régions est mesurée par le degré de synchronisme entre les activités cérébrales de ces régions. Dans l'étude décrite ci-dessus, l'activité cérébrale et la connectivité fonctionnelle étaient mesurées par électroencéphalogramme (દદG) (Figure IB).

\section{L'analyse des réseaux de connectivité} par la théorie des graphes

L'ensemble des régions du cerveau et de ses connexions forme un réseau complexe dans lequel les informations issues de régions spécialisées peuvent être intégrées [6]. Ces réseaux sont mathématiquement représentés par des graphes constitués de nœuds (représentant les régions du cerveau) reliés par des arêtes (représentant les connexions entre les régions) (Figure 1C).

Récemment, la théorie des graphes a été introduite en neurosciences pour caractériser les réseaux complexes du cerveau. Cette théorie permet, entre autres, de mesurer la résilience, qui quantifie l'effet sur le réseau de la suppression d'un nœud. Un réseau a une grande résilience si ses propriétés sont peu modifiées par la suppression du nœud. À titre de comparaison, le réseau aérien présente une grande résilience car il est souvent possible de trouver un chemin alternatif lorsqu'un aéroport est fermé.

\section{Redondance et déconnexion chez} les enfants atteints d'autisme Une augmentation significative de la résilience a été observée dans le cerveau des 


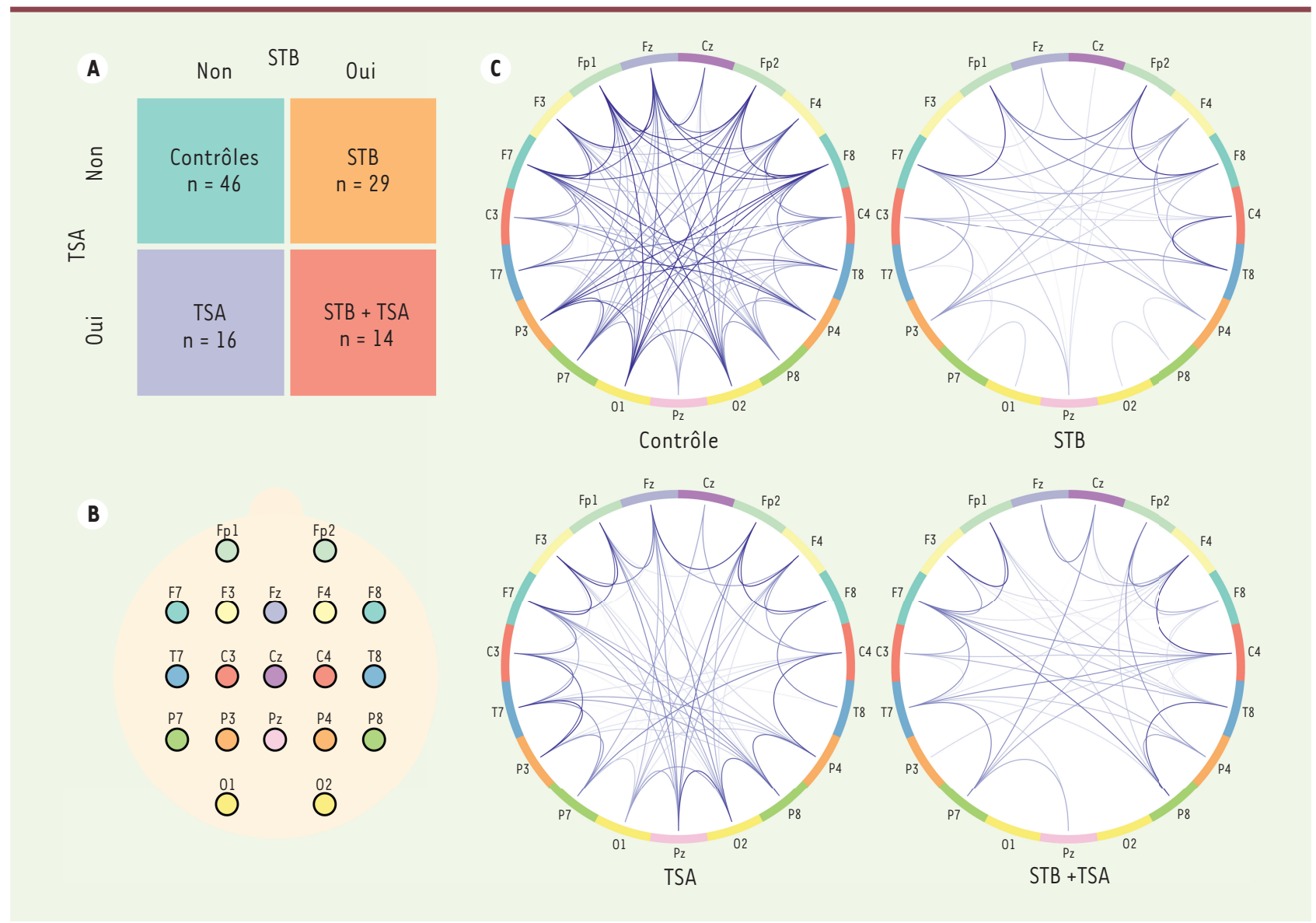

Figure 1. Analyse de la connectivité fonctionnelle chez les patients atteints de TSA. A. La population étudiée est structurée par deux facteurs : le diagnostic ou non d'une sclérose tubéreuse de Bourneville (STB) et le diagnostic ou non de troubles du spectre autistique (TSA). Cette structure permet d'identifier les effets spécifiques aux TSA et à la STB. B. Localisation des électrodes \&દG par le système international $10-20^{1}$. Chaque électrode représente un nœud du réseau. $C$. Illustrations des réseaux de connectivité fonctionnelle d'un sujet aléatoirement choisi dans chacun des quatre groupes. Reproduit de [4]. Fp : frontal polar ; F : frontal ; T : temporal ; C : central ; P : pariétal ; 0 : occipital.

patients atteints d'autisme (syndromique et non syndromique) (Figure 2). Deux explications plausibles de cette observation peuvent être avancées, reflétant des aspects différents des mécanismes neurobiologiques de l'autisme. Une augmentation de la résilience pourrait être la conséquence d'une redondance des connexions. En présence de connexions redondantes, l'information peut en effet trouver des voies secondaires pour se propager lorsqu'un nœud est supprimé. Cette redondance de connexions a cependant un

${ }^{1}$ Le système international 10-20 est une méthode permettant de déterminer l'emplacement d'électrodes au niveau du cuir chevelu dans le cadre d'un દદG. Il permet la normalisation des études et la comparaison des દદG entre individus. Le 10 et le 20 fait référence au fait que les distances réelles entre les électrodes adjacentes sont soit $10 \%$ soit $20 \%$ de la distance totale avant-arrière ou droite-gauche du crâne. coût métabolique important et le cerveau cherche constamment un compromis entre minimiser ce coût et maximiser l'efficacité topologique [7]. Physiologiquement, la redondance de connexions chez les enfants atteints d'autisme pourrait être expliquée par un «élagage » imparfait des connexions pendant la période de développement [9], en accord avec des études réalisées au niveau moléculaire et cellulaire chez les patients autistes.

Une augmentation de la résilience pourrait aussi impliquer une diminution de la spécialisation fonctionnelle des régions du cerveau. En effet, un excès de résilience caractérise un système biologique dégénéré dans lequel des éléments structurellement différents peuvent assurer la même fonction. Puisque le réseau continue de fonctionner de la même façon avant et après suppression d'un de ses nœuds, la présence de ces derniers semble moins critique, ce qui suggère que leur fonction est moins spécifique.

Par ailleurs, l'étude a également montré que les réseaux de connectivité des patients atteints d'autisme sont caractérisés par une diminution significative du rapport entre la connectivité à longue distance et la connectivité à courte distance. Comme la connectivité moyenne ne diminue pas, cette observation suggère que les cerveaux des patients atteints d'autisme présentent une sousconnectivité à longue distance et une sur-connectivité à courte distance. Cette observation est en faveur du modèle du syndrome de déconnexion et lui offre, 


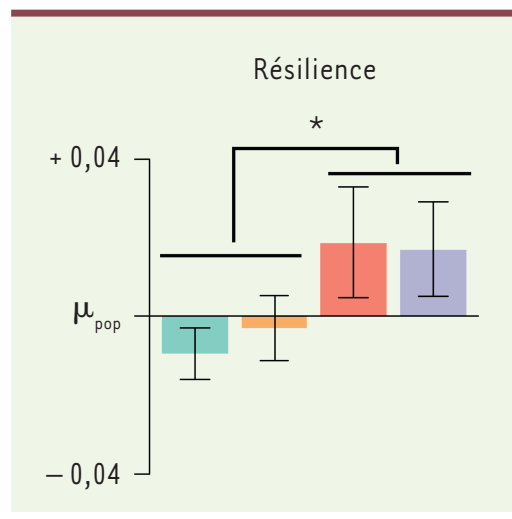

pour la première fois, une validation croisée chez les patients atteints d'autisme syndromique et non syndromique.

\section{Conclusion}

L'étude des réseaux de connectivité fonctionnelle cérébrale chez les patients atteints d'autisme syndromique et non syndromique a démontré des mécanismes neurobiologiques communs aux deux étiologies. Les patients atteints d'autisme présenteraient une diminution des connexions à longue distance au profit d'une augmentation et d'une redondance des connexions à courte distance. Ce réseau pourrait réagir de façon moins spécifique à différents stimulus et entraverait la réalisation de tâches cognitives complexes nécessitant l'intégration d'informations issues de régions corticales éloignées. Ces interprétations pourraient représenter la base neuro-
Rapport entre connectivité à longue et courte distance

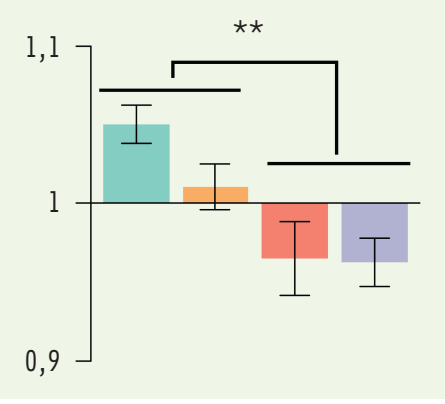

biologique du profil cognitif typique des patients atteints d'autisme. $\diamond$

The brain functional network of children with autism: redundancy and disconnection

\section{LIENS D'INTÉRÊT}

JMP is paid on a consultant basis for the Novartis EXIST-III trial: A study of the efficacy and safety of two trough-ranges of everolimus as adjunctive therapy in patients with tuberous sclerosis complex (TSC).

\section{REMERCIEMENTS}

Maxime Taquet est aspirant du FRS-FNRS. Ce travail a été financé en partie par le National Institute of Health (P20 RFA-NS-12-006, IU0INS082320-01). JMP reçoit un financement de la Faculty Development Fellowship sous la «Eleanor and Miles Shore $50^{\text {th }}$ Anniversary Fellowship Program for Scholars in Medicine», Boston Children's Hospital, Department of Neurology.
Figure 2. Réseaux de connectivité chez les patients atteints d'autisme. Les réseaux de connectivité fonctionnelle des patients atteints d'autisme (syndromique ou non) présentent une résilience accrue (gauche) et un rapport plus faible entre la connectivité à longue distance et à courte distance (droite) par rapport aux deux groupes contrôles.
Contrôles

STB

STB + TSA

TSA
Haute autorité de santé. Autisme et autres troubles envahissants du développement, Argumentaire: janvier 2010.

2. Just MA, Cherkassky VL, Keller TA, Minshew NJ. Cortical activation and synchronization during sentence comprehension in high-functioning autism: evidence of underconnectivity. Brain Oxford Univ Press 2004 ; 127 : 1811-21.

3. Deen B, Pelphrey K. Perspective: Brain scans need a rethink. Nature $2012 ; 491$ : S20.

4. Peters JM, Taquet M, Vega C, et al. Brain functional networks in syndromic and nonsyndromic autism: a graph theoretical study of દ๕G connectivity. BMC Med $2013 ; 11: 54$.

5. Tye C, Bolton P. Neural connectivity abnormalities in autism: Insights from the tuberous sclerosis model. BMC Med $2013 ; 11: 55$.

6. Sporns 0, Bullmore $\varepsilon$. Complex brain networks: graph theoretical analysis of structural and functional systems. Nat Rev Neurosci 2009 ; 10 : 186-98.

7. Bullmore $\varepsilon$, Sporns 0 . The economy of brain network organization. Nat Rev Neurosci 2012 ; 13 : 336-49.

8. Thiebaut de Schotten M. L'étude des connexions cérébrales révèle les bases anatomiques de la dominance de l'hémisphère droit pour l'attention spatiale. Med Sci (Paris) $2012 ; 28: 21-3$.

9. Letellier M, Sherrard RM, Lohof AM, Marian J. Faire et refaire une synapse en choisissant un partenaire. Une question d'étiquette pour les neurones ? Med Sci (Paris) $2010 ; 26: 724-8$.

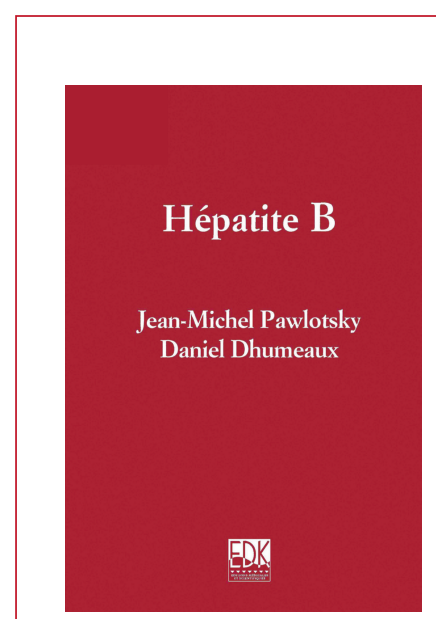

ISBN : 978-2-8425-4131-6 576 pages

\section{Bon de commande}

À retourner à EDK, 25, rue Daviel - 75013 Paris

Tél. : 0158101905 - Fax : 0143293262 - E-mail : edk@edk.fr

NOM :

Prénom :

Adresse :

Code postal :

Ville :

Pays :

Fonction :

Je souhaite recevoir l'ouvrage Hépatite B : $54 €+3 €$ de port $=\mathbf{5 7} €$ TTC offre exceptionnelle réservée aux abonnés à $\mathrm{m} / \mathrm{s}$ jusqu'au 31 décembre 2010

en ................ exemplaire, soit un total de ..................................... €

$\square$ Par chèque, à l'ordre de $\mathbf{E} \mathbf{D} \mathbf{K}$

$\square$ Par carte bancaire : $\square$ Visa $\square$ Eurocard/Mastercard

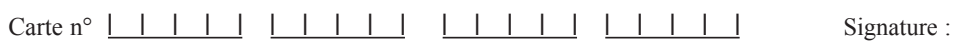

Date d'expiration: $\quad \underline{1}|\underline{1}|$

$\mathrm{N}^{\circ}$ de contrôle au dos de la carte : $\quad$ ～～～～～～| 